\title{
Lidil
}

Revue de linguistique et de didactique des langues

Sémantique des noms et adjectifs d'émotion

\section{Les noms de sentiment}

Esquisse de typologie sémantique fondée sur les collocations verbales

\section{Vannina Goossens}

\section{(2) OpenEdition}

\section{Journals}

Édition électronique

URL : http://journals.openedition.org/lidil/102

DOI : $10.4000 /$ lidil. 102

ISSN : 1960-6052

Éditeur

UGA Éditions/Université Grenoble Alpes

\section{Édition imprimée}

Date de publication : 1 décembre 2005

Pagination : 103-121

ISBN : 2-914176-13-9

ISSN : $1146-6480$

Référence électronique

Vannina Goossens, "Les noms de sentiment », Lidil [En ligne], 32 | 2005, mis en ligne le 05 octobre 2007, consulté le 01 mai 2019. URL : http://journals.openedition.org/lidil/102 ; DOI : 10.4000/lidil.102

Ce document a été généré automatiquement le 1 mai 2019.

(C) Lidil 


\title{
Les noms de sentiment
}

\author{
Esquisse de typologie sémantique fondée sur les collocations verbales
}

\author{
Vannina Goossens
}

1 Les noms de SENTIMENT' (désormais N_SENT) - tels colère, amour, joie, tristesse ou honte présentent une structure sémantique complexe. En effet, ils ne semblent réguliers ni du point de vue de leurs caractéristiques aspectuelles, ni du point de vue de leur structure actancielle. Peut-on néanmoins fonder une typologie établissant des sous-catégories cohérentes de N_SENT ? Les classifications du lexique des SENTIMENTS existantes (Galati et Sini, 1998, 2000; Johnson-Laird et Oatley, 1989) étant plutôt basées sur des critères cognitifs, elles ne prennent guère en compte les caractéristiques linguistiques des lexèmes considérés, en particulier leur combinatoire lexicale. Notre objectif ${ }^{2}$ ici est donc d'explorer les possibilités de fonder une typologie des N_SENT du français qui reflète leur fonctionnement linguistique. L'approche choisie se fonde sur une étude systématique des co-occurrents de ces noms, ou collocations, dans le sens donné à ce terme par Igor Mel'čuk $(1995,1998)$ ou encore Agnès Tutin et Francis Grossmann (2002). Chez ces auteurs, les collocations ne sont pas vues uniquement comme des mots qui tendent statistiquement à apparaître ensemble mais plutôt comme des cooccurrences lexicales privilégiées liant deux éléments linguistiques qui entretiennent une relation syntaxique et sémantique particulière. Les collocatifs, en tant que co-occurrents privilégiés, nous renseignent ainsi sur les caractéristiques sémantiques des noms auxquels ils s'associent. La perspective adoptée n'est pas purement distributionnelle : le but est bien, à partir de la description de la combinatoire lexicale, de mieux cerner les contenus sémantiques des N_SENT pour permettre leur classement.

2 Étant donné la difficulté de la tâche, un point de vue particulier a été privilégié, celui d'appréhender les noms de SENTIMENT à partir de leur structure actancielle et des dimensions sémantiques qui lui sont liées. Ces noms étant prédicatifs, la prise en compte de leur structure actancielle se révèle en effet un préalable indispensable, bien que non suffisant pour les discriminer aux plans sémantique et syntaxique: il est nécessaire d'examiner également l'expression de la source du SENTIMENT (cause extérieure ou volonté de l'expérienceur'), le "pouvoir » que le SENTIMENT a sur son expérienceur et la possibilité qu'a ou non ce dernier de le contrôler. D'autres dimensions, qui jouent sans 
doute également un rôle important dans l'optique typologique qui est la nôtre, ont pour l'instant été laissées de côté, soit en raison de leur grande complexité (c'est le cas pour l'aspect), soit parce qu'elles nous ont semblé, à tort ou à raison, moins pertinentes (l'intensité du N_SENT et ses manifestations). La centration sur la structure actancielle présente également l'intérêt d'être complémentaire des études effectuées jusqu'à présent qui ont souvent privilégié la description de leurs caractéristiques aspectuelles (Anscombre, 1995, 1996 ; Flaux et Van de Velde, 2000 ; Tutin et al., à paraître).

Définition de la classe des noms de SENTIMENT

Définir une classe des N_SENT n'est pas chose aisée mais c'est un préalable indispensable à notre travail. La coexistence des termes émotion et sentiment mais également la complexité sémantique et la polysémie de ces noms font qu'il est malaisé de les regrouper dans un même champ sémantique et de nommer ce dernier. Nos critères de définition de la classe reprennent certains de ceux qui sont classiquement mentionnés dans la littérature sur le sujet (Anscombre, 1995, 1996; Balibar-Mrabti, 1995; Flaux et Van de Velde, 2000 ; Leeman, 1995 ; Tutin et al., à paraître'). Sémantiquement, les N_SENT renvoient à un processus psychologique. Le premier critère d'appartenance à cette classe est la compatibilité de ces noms avec les verbes supports éprouver et ressentir. De nombreuses critiques peuvent cependant être formulées à l'égard de ce critère. Tout d'abord, sa compatibilité avec les N_SENT n'est pas toujours évidente. Par ailleurs, il ne permet pas de distinguer les N_SENT des noms de sensation. Ceux-ci se distinguent pourtant sémantiquement par l'absence de la dimension psychologique au profit de la seule dimension "physique ». Plus généralement, l'incompatibilité de la distribution de ces deux verbes avec des noms que nous considérons intuitivement comme n'étant pas des N_SENT n'est pas toujours évidente. Ce critère n'étant donc pas suffisant à lui seul, nous y adjoignons la compatibilité avec un sentiment de. Ce deuxième critère nous permet de distinguer les N_SENT (peur, colère, amour, mépris...) des noms de sensation (faim, soif...) qui s'accommodent du premier critère mais pas du second (*un sentiment de faim, de soif). La polysémie de ces noms fait que certains d'entre eux peuvent relever à la fois des N_SENT et des noms de sensation (douleur, fatigue), des N_SENT et des noms de qualité (gaieté, tristesse), etc. Nous ne nous sommes ici intéressée qu'à l'acception SENTIMENT de tous ces termes. Enfin, nous distinguons entre noms de sentiment (désormais $\mathrm{N}_{-}$sent) et noms d'émotion (désormais N_émotion) à l'intérieur de la classe générale des N_SENT. Cette distinction est basée sur les caractéristiques actancielles de ces noms, caractéristiques que nous allons aborder dans la partie qui suit.

4 Nous avons choisi d'étudier la classe des N_SENT au travers de trente noms ${ }^{5}$ qui nous en semblent de bons représentants. Pour les sélectionner nous nous sommes basée sur leur fréquence (dans Frantext) mais également sur les propositions d'organisation du lexique des SENTIMENTS comme celles de Philip N. Johnson-Laird et Keith Oatley (1989) ou de Dario Galati et Barbara Sini $(1998,2000)$, ainsi que sur des travaux non-linguistiques portant sur les SENTIMENTS eux-mêmes ${ }^{6}$ qui nous ont amenée à sélectionner des noms de fréquence moins importante mais véhiculant des caractéristiques sémantiques spécifiques qui méritaient d'être étudiées afin d'avoir une vision la plus large possible de ce champ.

MéthodologieConstruction du corpus

5 La méthodologie qui nous a semblé la plus appropriée à l'étude des collocations des $\mathrm{N}_{-}$ SENT est un travail sur corpus. En effet, le phénomène collocatif est un phénomène vivant, qui nécessite d'être étudié au travers d'occurrences attestées. Nous avons donc construit 
un corpus à partir de la base catégorisée de Frantext en sélectionnant les romans' de 1950 à 2005 (197 textes), ce qui correspond à un corpus de plus de 16 millions de mots. Nous y avons recherché toutes les collocations verbales auxquelles donnaient lieu nos trente $\mathrm{N}_{-}$ SENT. Une fois éliminées les acceptions non pertinentes des noms polysémiques, nous aboutissons à un peu plus de 8000 collocations. Le nombre de collocations pour chaque $\mathrm{N}_{-}$ SENT est cependant très disparate. Ainsi, peur a produit plus de trois mille collocations alors que des noms comme frayeur ou effroi n'en ont produit qu'une trentaine. C'est pour cette raison, mais aussi parce que l'examen d'un corpus même vaste ne dispense pas d'une réflexion sur l'acceptabilité - que nous n'excluons pas le recours à l'intuition pour vérifier la grammaticalité. En cas de doute, nous avons eu également recours à des exemples attestés, mais ne figurant pas dans le corpus principal. La disparité des fréquences des collocations rencontrées dans le corpus rend difficile un traitement statistique valide et notre approche restera de ce fait essentiellement qualitative. Nous fournirons cependant la fréquence des collocations relevées, lorsque cette information sera utile.

Choix des paramètres pris en compte pour l'élaboration de notre typologieLa structure actancielle des noms de SENTIMENT

6 Nous nous sommes inspirée des travaux d'Igor Mel'čuk (Mel'čuk et alii, 1995 ; Mel'čuk et Wanner, 1996; Mel'čuk (dir.), 1984, 1988, 1992 et 1999) pour analyser la structure actancielle des N_SENT.

7 Les N_SENT ont deux ou trois actants sémantiques. Leur premier actant est humain et obligatoire. Il désigne la personne qui éprouve le SENTIMENT exprimé (Paul dans Paul a peur ): c'est l'expérienceur, lorsque la personne subit ce SENTIMENT, l'agent, lorsque la personne est « active » et participe à la construction du SENTIMENT. Ces noms ont également une cause, facultative, qui désigne l'élément qui déclenche la survenue du SENTIMENT chez l'expérienceur (Marie dans Marie fait peur à Paul) et/ou un objet, facultatif également, qui désigne la personne vers qui est dirigée le SENTIMENT (Claire dans Luc ressent de l'amour pour Claire). L'objet est généralement humain (le respect de Paul envers Marie [objet]) alors que la cause peut aussi bien renvoyer à un humain qu'à un non-humain (Paul [cause] fait peur à Luc, la peur du noir [cause], la peur de mourir [cause] etc.).

8 La structure actancielle nous permet de distinguer, à l'intérieur des N_SENT, les N_sent des N_émotion. Les N_sent ont obligatoirement au moins deux actants : leur premier actant s'apparente plus à un agent qu'à un expérienceur et leur second actant est un objet, objet qui est obligatoirement lexicalisé lorsque ces noms sont employés avec un verbe support tel que avoir, éprouver ou ressentir: *j'ai de l'amour, de la haine mais j'ai de l'amour, de la haine pour Pierre, ${ }^{*} j$ e ressens du respect, du mépris mais je ressens $d u$ respect, $d u$ mépris pour Paul etc. Certains N_sent ont également un troisième actant cause. Par exemple, mépris possède à la fois un expérienceur/agent, une cause et un objet ( $L u c$ éprouve du mépris pour Paul du fait de sa lâcheté) contrairement à amour qui est bi-actanciel : il possède un expérienceur/agent et un objet mais pas de cause (Paul éprouve de l'amour pour Marie mais *Paul éprouve de l'amour pour Marie à cause de sa gentillesse). Les N_émotion ont également au moins deux actants: un expérienceur/agent et une cause, même si celle-ci n'est pas obligatoirement lexicalisée : j'ai peur (du noir). Contrairement aux N_sent, la plupart des N_émotion n'ont pas d'objet (peur, joie, tristesse, surprise...) et quand ils en ont un, celui-ci peut ne pas être lexicalisé : je suis en colère (contre toi), j'éprouve de la rage, de la fureur (envers Paul). 
9 En observant notre corpus nous remarquons qu'au plan syntaxique, ces actants sont réalisés de différentes façons.

10 - L'agent/expérienceur est quasiment toujours réalisé : en position sujet (Paul a peur, Paul a peur de Luc/du noir) ou en position complément (Luc fait peur à Paul, la peur envahit Paul ou encore, dans cet exemple du corpus le gêneur [...] méritait leur compassion $\left.{ }^{8}\right)$. Cette réalisation n'est cependant pas obligatoire, en témoignent des exemples comme bientôt la peur monta', ou : Olga était [...] une réfugiée qui méritait de la compassion ${ }^{10}$.

11 - La cause est fréquemment réalisée par un SN en position sujet : son monocle d'étoffe noire me fit peur", mais également lorsque c'est l'expérienceur qui est en position sujet : Paul a peur de Luc. Elle peut théoriquement être également réalisée lorsque le N_SENT est en position sujet (du type la peur monte en Paul à cause de l'obscurité) ou lorsque c'est l'objet du N_SENT qui est en position sujet (comme dans Laura mérite le respect de Paul pour son courage ), mais dans les faits ces réalisations sont quasi inexistantes.

12 - L'objet est pratiquement toujours réalisé lorsque l'expérienceur est en position sujet (dans des structures du type Paul éprouve de l'amour pour Laura) mais ce n'est pas systématique, il peut être uniquement sous-jacent : Paul est en colère (contre Laura). Il est également réalisé lui-même en position sujet : Laura mérite le respect. Dans les autres cas, il est sous-jacent comme dans l'amour (pour Laura) envahit Paul.

Paramètres sémantiques liés aux collocations verbales

13 Nous avons procédé à une analyse sémantique des collocatifs relevés afin de dégager ceux qui expriment la source du SENTIMENT, le pouvoir qu'a ce SENTIMENT sur son expérienceur et la possibilité qu'a ce dernier de le contrôler. Nous avons distingué, à partir de l'étude de près de 2000 collocations, quatre dimensions sémantiques véhiculées par les collocatifs verbaux permettant d'éclairer notre problématique :

14 - la « causation » : les verbes caractérisés par ce trait expriment le fait qu'un événement, quelqu'un ou quelque chose amènent une personne à ressentir un SENTIMENT (faire peur, donner du bonheur, causer de la peine, etc.). Notons que le sujet de ces verbes peut être soit l'actant cause (Le noir fait peur à Paul), ce qui est généralement le cas, soit parfois l'actant objet (Paul inspire de la compassion à Pierre).

15 - la «volition»: les verbes caractérisés par cette dimension véhiculent l'idée que l'expérienceur du SENTIMENT est en quelque sorte un agent et qu'il décide plus ou moins d'éprouver un SENTIMENT (se mettre en colère, vouer de la haine, etc.).

16 - la «possession": certains verbes expriment le fait que le SENTIMENT éprouvé par l'expérienceur a un certain pouvoir sur celui-ci, que ce dernier le subit (la colère envahit, la joie remplit, etc.).

17 - le « contrôle » : certains verbes expriment la capacité que l'expérienceur a de contrôler le SENTIMENT qu'il éprouve ou bien ses manifestations (surmonter sa peur, s'abandonner au désespoir, ne pas cacher sa joie, etc.). La différence entre cette dimension et la précédente est une question de point de vue: le contrôle est appréhendé du point de vue de l'expérienceur, la possession du point de vue du SENTIMENT.

18 Nous allons maintenant étudier comment les collocatifs porteurs de ces quatre dimensions sémantiques se combinent avec les trente N_SENT sélectionnés.

Étude des collocations verbales des noms de SENTIMENTCausation 
19 C'est la dimension la plus productive dans le corpus, elle représente environ 1200 collocations. Nous rencontrons différents types de causatifs :

20 - des causatifs neutres : $\mathrm{Y}^{12}$ (ou Z) inspire, impose, fait, cause, donne, apporte, procure, provoque N_SENT à X. Ils représentent quasiment l'intégralité des collocatifs causatifs (plus de 1100 collocations).

21 - des causatifs aspectuels : Y (ou Z) fait naître, engendre, réveille, chasse, achève, endigue N_SENT, plonge, jette $\mathrm{X}$ dans N_SENT.

22 - des causatifs intensifs : Y (ou Z) attise, excite, avive, augmente, apaise, calme N_SENT.

23 La grande majorité des N_émotion se combinent avec des verbes causatifs et en particulier des causatifs neutres qui sont les plus fréquents dans le corpus (faire peur, procurer du bonheur, mettre en joie, faire de la peine, mettre en colère...) et des causatifs aspectuels exprimant le début de l'émotion (éveiller la peur...). Le fait que ces noms possèdent un deuxième actant cause et que leur premier actant s'apparente plus à un expérienceur qu'à un agent (la cause du N_émotion est plutôt extérieure à l'expérienceur) peut expliquer ce phénomène. Un petit nombre de N_émotion apparaît cependant rarement avec des causatifs. C'est le cas de angoisse, désespoir, ennui et mélancolie, qui semblent dénoter des émotions n'ayant pas de cause précise, mais aussi de crainte qui dénote une attente plutôt qu'une réaction à un événement (Bresson et Dobrovol'skij, 1995).

24 Le premier actant des N_sent quant à lui semble revêtir davantage un rôle d'agent que celui des N_émotion et le second actant, la personne vers qui se dirige le sentiment, est davantage un objet sémantique qu'une cause. Les N_sent ont donné lieu à beaucoup moins de collocations que les N_émotion dans ce corpus, ce qui rend l'interprétation de leurs occurrences plus délicates. La moitié d'entre eux ont cependant produit des collocations assez nombreuses avec des causatifs. C'est le cas de dégoût (inspirer, causer), envie (inspirer, susciter), compassion (éveiller) et respect qui entre dans des collocations d'une nature particulière : inspirer le respect mais également imposer le respect, obliger, forcer au respect.

Les causatifs intensifs et les causatifs aspectuels exprimant les phases médiane et finale du SENTIMENT sont trop rares dans le corpus pour que l'on puisse en tirer des conclusions. De plus, ils n'expriment pas vraiment la cause du SENTIMENT qui est ce qui nous intéresse ici.

Volition

26 Nous rencontrons la dimension de volition avec des collocatifs du type : X se met en, prend en, tient en, nourrit, voue, porte N_SENT. Ceux-ci ne se sont combinés qu'avec des N_SENT dirigés vers un objet, ce qui semble cohérent, puisque le premier actant de ces noms (qu'il s'agisse de N_sent ou de N_émotion) s'apparente plus à un agent qu'à un expérienceur. Cette dimension est cependant très peu fréquente parmi les collocatifs verbaux de ce corpus : nous n'en relevons qu'une centaine. Peu de verbes nous apparaissent pouvoir la véhiculer et comme ils se rapportent à des noms peu fréquents (les N_sent en particulier), il est difficile de considérer que ces associations sont véritablement significatives. Cellesci sont cependant très intéressantes à observer et, même si on ne peut pas en tirer de conclusions définitives, elles sont révélatrices de tendances qui mériteraient d'être étudiées de manière plus approfondie. 
27 Colère, rage et fureur ont produit de nombreuses occurrences avec se mettre en. Ces collocations sont différentes de mettre en colère, construction dans laquelle une cause extérieure à l'expérienceur était explicitement mentionnée. Dans le cas de se mettre en colère, même si on ne nie pas le rôle d'une cause extérieure dans la survenue du SENTIMENT de colère, la décision de se mettre en colère semble venir de l'expérienceur lui-même, qui se présente ainsi en tant qu'agent du SENTIMENT.

28 Parmi les N_sent, seuls dégoût, haine et mépris se combinent avec des collocatifs exprimant la dimension de volition : prendre en haine, nourrir, vouer de la haine, prendre en dégoût, tenir en mépris... Envie, respect et compassion n'ont donné lieu à aucune collocation de ce type, ce qui n'est peut-être pas anodin puisque ces trois noms tendaient à s'associer avec des causatifs. Jalousie et amour ne semblent être ni des N_sent causés, ni des N_sent voulus.

Possession

29 La possession est exprimée par des collocations du type : N_SENT envahit, saisit, cloue, frappe, prend X, où le N_SENT est en position sujet, ou bien des structures passives du type $\mathrm{X}$ est saisi de N_SENT. Cette dimension est relativement fréquente dans notre corpus avec plus de 400 collocations. ces collocatifs se sont pour l'essentiel associés avec des N_émotion et parmi ceux-ci des noms exprimant des émotions intenses : la peur, l'angoisse, la panique envahissent, prennent, saisissent, submergent, la terreur envahit, la joie, le bonheur inondent, s'emparent de, la tristesse envahit, pénètre, submerge, le désespoir s'empare de, envahit, la colère, la rage, la fureur envahissent, prennent, gagnent, la honte, le dégoût envahissent, prennent, submergent... Les noms exprimant des émotions moins intenses comme crainte, gaieté, peine, ennui ou mélancolie n'en ont pas ou quasiment pas produit. Il en est de même pour la majorité des N_sent, même intenses, ce qui ne paraît pas étonnant du fait qu'ils prennent plutôt leur source dans le sujet lui-même qui est par là même plus un agent qu'un expérienceur. Stupeur, surprise, frayeur et effroi semblant pourtant dénoter des émotions intenses, n'ont pas ou peu donné lieu à de telles collocations. Des collocations du type cloué de surprise se rencontrent cependant dans d'autres contextes ${ }^{13}$, ce qui nous amène à penser qu'il s'agit peut-être d'un phénomène lié à la taille de notre corpus. Nous nous limiterons cependant, pour l'instant en tout cas, aux résultats obtenus avec ce corpus pour bâtir notre typologie.

Contrôle

30 Comme Agnès Tutin et al. (à paraître), nous distinguons entre deux types de contrôle dans les collocations de notre corpus :

31 - le contrôle du SENTIMENT en tant que tel (environ 200 collocations) : X ravale, boit, surmonte, domine, vainc, dompte, tue, oublie, chasse, rit de, se moque de, ou bien accepte, laisse monter, cède à, laisse déferler son N_SENT etc.

- et le contrôle de ses manifestations : X dissimule, cache, ou bien ne peut contenir, laisse éclater son N_SENT ${ }^{14}$.

33 Seuls les N_SENT ayant donné lieu à des collocations avec des verbes exprimant la possession ont produit des collocations avec les verbes exprimant le contrôle. Nous pouvons expliquer cela par le fait que les N_SENT susceptibles d'être contrôlés (ou de donner lieu à une perte de contrôle) sont ceux qui envahissent, submergent l'individu de l'extérieur. Ainsi, les N_sent ne se sont quasiment pas associés aux collocatifs exprimant le contrôle dans le sens où ceux-ci prennent plutôt leur source dans le sujet lui-même. 

possession n'ont pas donné lieu à des collocations avec des verbes exprimant le contrôle. Certains N_SENT semblent ainsi prendre possession de leur expérienceur sans que celui-ci ne puisse réagir : c'est le cas de tristesse, joie, bonheur et dégoût par exemple. Ceci peut être interprété comme une impossibilité de contrôle ou bien un refus de contrôle (pour bonheur par exemple, du fait de sa polarité positive). Angoisse et désespoir ont produit des collocations qui expriment le fait que l'expérienceur tente de contrôler son émotion ( lutter contre, résister au désespoir...) ou au moins de la modérer (calmer son angoisse) mais surtout que l'individu n'arrive pas ou renonce à contrôler son émotion (céder à, laisser déferler l'angoisse, s'abandonner à, se laisser aller à céder au désespoir).

autres noms ayant donné lieu à des collocations avec les verbes exprimant la possession (à savoir peur, panique, terreur, colère, rage, fureur, chagrin et honte) ont produit des collocations, souvent métaphoriques, exprimant le contrôle du SENTIMENT. Les collocatifs sont très variés : l'individu "apprivoise » son SENTIMENT (dompter, dominer, surmonter sa peur), il l'éradique (vaincre, tuer sa peur, noyer son chagrin), échappe à son emprise (échapper à la panique, s'arracher à la terreur), le combat (combattre sa peur, lutter contre sa peur, la terreur), l'empêche de prendre de l'ampleur (ravaler sa fureur, sa rage, sa colère, contenir sa colère, sa fureur, refouler, réprimer sa colère), s'en moque (se moquer, rire de sa peur), ainsi que quelques collocatifs spécifiques à un N_SENT (boire sa honte).

Typologie

tre étude nous permet d'élaborer une typologie des N_SENT qui reflète leurs propriétés combinatoires les plus discriminantes. Nous avons choisi comme critère de départ de prendre en compte à la fois l'existence de l'actant objet et la présence de collocations formées avec des verbes véhiculant la dimension de causation. Ce premier aspect permet d'opposer N_SENT orienté vers un objet vs N_SENT non orienté vers un objet et N_SENT causé vs N_SENT non causé. Les collocations formées avec un verbe causatif nous renseignent plus sur l'importance de la cause du N_SENT que sur l'existence d'un actant cause pour ce nom. Nous avons ainsi classé un nom tel que angoisse dans les N_SENT non causés bien qu'il possède un actant cause. Nous préciserons pour chacune des classes l'adéquation ou l'inadéquation du critère de causatif avec l'existence d'un actant cause. Nous avons ensuite sélectionné trois critères secondaires spécifiquement sémantiques. Le premier critère traduit ce que nous avons appelé "possession » et correspond à la situation dans laquelle le SENTIMENT est un agent qui « envahit » l'expérienceur ; le second marque la situation dans laquelle le SENTIMENT est contrôlé par l'expérienceur; enfin le troisième, la "volition ", marque le fait que l'expérienceur ne subit pas passivement le SENTIMENT mais l'assume et le met en œuvre activement. Ces différents critères aboutissent à une classification composée de quatre grandes classes de noms regroupant dix sous-classes au total.

A) Les N_SENT causés et non dirigés vers un objet : peur, terreur, panique, joie, tristesse, bonheur, peine, gaieté, chagrin, stupeur, surprise, effroi, frayeur. Ces noms s'associent avec des collocatifs causatifs (inspirer, causer, faire, semer...), possèdent un actant cause et ne sont pas dirigés vers un objet.

a) Ceux qui peuvent marquer la possession aussi bien que le contrôle : peur, terreur, panique, chagrin, joie. Les noms qui entrent dans cette classe s'associent à la fois à des collocatifs porteurs de la dimension de possession (la peur, la terreur, la joie envahissent, saisissent)... X, la joie envahit, inonde, remplit... X) et des collocatifs 
porteurs de la dimension contrôle (X domine, dompte, surmonte, combat sa peur, cède à, échappe à la panique, lutte contre, contrôle sa terreur, retient, contient, maîtrise sa joie).

b) Ceux qui admettent la possession mais pas le contrôle : tristesse, bonheur. Les noms qui entrent dans cette classe s'associent à des collocatifs porteurs de la dimension possession (le bonheur envahit, s'empare de $\mathrm{X}$, la tristesse pénètre, envahit, submerge X) mais n'acceptent pas de se combiner avec des collocatifs porteurs de la dimension contrôle.

c) Ceux qui n'admettent ni la possession ni le contrôle : peine, gaieté, stupeur, surprise, effroi, frayeur ${ }^{15}$. Les noms qui entrent dans cette classe ne se combinent ni avec des collocatifs porteurs de la dimension possession ni avec des collocatifs porteurs de la dimension contrôle.

B) Les N_SENT non causés et dirigés vers un objet : haine, mépris, jalousie, amour. Ces noms sont dirigés : l'objet du N_SENT est obligatoirement présent ou au moins sous-jacent. Par contre, ils n'acceptent pas de s'associer à des collocatifs causatifs même s'ils possèdent un actant cause (à l'exception de amour qui n'a pas d'actant cause).

a) Ceux qui traduisent le fait que le SENTIMENT est voulu, ou tout au moins assumé par l'expérienceur : haine, mépris. Les termes qui entrent dans cette classe s'associent à des collocatifs exprimant la volition (prendre en haine, tenir en mépris).

b) Ceux qui traduisent le fait que le SENTIMENT est subi par l'expérienceur : jalousie, amour. Les termes qui entrent dans cette classe n'acceptent pas de se combiner à des collocatifs exprimant la volition.

39 C) Les N_SENT causés et dirigés vers un objet : honte, dégoût, colère, rage, fureur, envie, respect, compassion. Ces noms sont dirigés, ils s'associent à des collocatifs causatifs ( inspirer, causer, faire, semer...) et possèdent un actant cause.

a) Ceux qui admettent la possession mais ne sont pas «voulus" par l'expérienceur: honte, dégoût. Les termes qui entrent dans cette classe se combinent avec des collocatifs porteurs de la dimension possession (la honte, le dégoût envahissent, submergent, prennent $\mathrm{X}$ ).

b) Ceux qui admettent la possession et qui sont "voulus" ou en tous cas assumés par l'expérienceur : colère, rage, fureur. Les termes qui entrent dans cette classe s'associent à la fois avec des collocatifs porteurs de la dimension possession (la colère, la fureur envahissent, prennent, gagnent $X$, la rage saisit, prend, s'empare de X) et avec des collocatifs exprimant la volition (se mettre en colère, en rage, en fureur).

c) Ceux qui n'admettent ni la possession ni la volition : envie, respect, compassion. Les termes qui entrent dans cette classe n'acceptent ni de s'associer à des collocatifs porteurs de la dimension possession ni à des collocatifs exprimant la volition.

D) Les N_SENT ni causés ni dirigés vers un objet : angoisse ${ }^{16}$, désespoir, crainte, ennui, mélancolie. Ces noms ne sont pas dirigés et ne s'associent pas avec des collocatifs causatifs même s'ils possèdent un actant cause.

a) Ceux qui admettent la possession : angoisse, désespoir. Les termes qui entrent dans cette classe se combinent à des collocatifs porteurs de la dimension possession (l'angoisse envahit, prend, saisit $\mathrm{X}$, le désespoir s'empare, prend, envahit $\mathrm{X}$ ).

b) Ceux qui n'admettent pas la possession : crainte, ennui, mélancolie. Les termes qui entrent dans cette classe n'acceptent pas de se combiner avec des collocatifs porteurs de la dimension possession.

41 Cette typologie permet ainsi de décrire les caractéristiques à la fois sémantiques et formelles des N_SENT que nous avons sélectionnés ainsi que les relations qu'ils entretiennent dans la classe des N_SENT. Elle se présente comme purement descriptive, même si nous pourrions, à terme, nous demander dans quelle mesure il est possible de trouver des hypothèses explicatives aux regroupements de noms que nous avons établis, 
afin de rechercher des éléments de convergence, au moins partiels, entre notre typologie linguistique et des typologies issues d'approches extralinguistiques.

À l'issue de cette étude nous constatons que l'orientation méthodologique empruntée pour élaborer notre typologie des N_SENT conduit à des résultats intéressants. En effet, le choix de dégager le profil sémantique de ces noms en partant de leurs propriétés combinatoires a abouti à des critères définitoires et typologiques beaucoup moins arbitraires que ceux que nous aurions dégagés avec une démarche intuitive. Les classes de noms élaborées ainsi rassemblent des noms que nous n'aurions pas pensé à rapprocher préalablement mais qui s'avèrent partager des caractéristiques sémantiques et formelles cohérentes. L'étude de la structure actancielle des N_SENT s'est ainsi avérée indispensable mais non suffisante pour les distinguer. Les dimensions sémantiques liées à cette structure actancielle que nous avons mises en évidence (à savoir la causation, la volition, la possession et le contrôle) se sont quant à elles révélées très pertinentes dans cette optique typologique. L'étude d'autres dimensions sémantiques véhiculées par les collocations des N_SENT comme celle de l'aspect permettrait d'affiner cette typologie. De même, une validation statistique de notre typologie à partir d'un corpus plus imposant et plus varié (comportant un sous-corpus oral par exemple), prenant également en compte une liste plus importante de N_SENT, se révélerait sans doute fructueuse.

\section{BIBLIOGRAPHIE}

ANSCOMBRE, J.-C. (1995) : Morphologie et représentation événementielle : le cas des noms de sentiment et d'attitude, Langue française, $\mathrm{n}^{\circ}$ 105, Grammaire des sentiments, Paris, Larousse, 40-54.

_, (1996) : Noms de sentiment, noms d'attitude et noms abstraits, in Les noms abstraits, histoire et théories, Flaux, N., Glatigny, M. et Samain, D. (eds.), Lille, Presses universitaires du Septentrion, 257-273.

BALIBAR-MRABTI, A. (1995) : Une étude de la combinatoire des noms de sentiment dans une grammaire locale, Langue française, 105, Grammaire des sentiments, Paris, Larousse, 88-97.

BRESSON, D. et DOBROVOL'SKIJ, D. (1995) : Petite syntaxe de la " peur ", Application au français et à l'allemand, Langue française, 105, Grammaire des sentiments, Paris, Larousse, 107-119.

COSNIER, J. (1994) : Psychologie des émotions et des sentiments, Paris, Retz.

DARWIN, C. (1872) : L'expression des émotions chez l'homme et les animaux (réimpression : Paris, Reinwald, 1980).

DESCARTES, R. (1649) : Les passions de l'âme (réimpression : Paris, Bibliothèque de la Pléiade, Gallimard, 1953).

DIRVEN, R. (1997) : Emotions as Cause and the Cause of Emotions, in The Language of Emotions: Conceptualization, Expression and Theoretical Foundation, Niemeier, S. and Dirven, R. (eds.), Amsterdam, John Benjamins, 55-83. 
FLAUX, N. et VAN DE VELDE, D. (eds) (2000) : Les Noms en français : esquisse de classement, Paris, Ophrys.

FRIJDA, N. H. (1988) : Les théories des émotions : un bilan, in Les émotions, Rime, B. et Scherer, K.R. (eds.) (1989), Neuchâtel-Paris, Delachaux et Niestlé, 21-72.

GALATI, D. et SINI, B. (1998) : Les mots pour dire les émotions : recherche sur la structure du lexique émotionnel italien, Revue de sémantique et de pragmatique, $\mathrm{n}^{\circ} 4$, Actualisation et détermination, Orléans, Presses Universitaires d'Orléans, 139-161.

-, (2000) : Les structures sémantiques du lexique français des émotions, in Les émotions dans les interactions, Plantin, C., Doury, M. et Traverso, V. (eds.), Lyon, Presses universitaires de Lyon, 75-87.

GROSS, M. (1995) : Une grammaire locale de l'expression des sentiments, Langue française, 105, Grammaire des sentiments, Paris, Larousse, 70-87.

GRosSmanN, F. et TUTIN, A. (à paraître) : Motivation of Lexical Associations in Collocations: the Case of Intensifiers denoting "Joy », à paraître dans Festchrift in Honour of Igor Mel'čuk, Wanner, L. (ed.), Amsterdam, John Benjamins.

JOHNSON-LAIRD, P. et OATLEY, K. (1989) : The Language of Emotions: An Analysis of a Semantic Field, in Cognition and Emotion, 3 (2), 81-123.

LEEMAN, D. (1991) : Hurler de rage, rayonner de bonheur : remarques sur une construction en de, Langue française, 91, Prépositions, représentations, référence, Paris, Larousse, 80-101.

-, (1995) : Pourquoi peut-on dire Max est en colère mais non *Max est en peur, Langue française, 105, Grammaire des sentiments, Paris, Larousse, 55-69.

MATHIEU, Y. (1999) : Les prédicats de sentiment, Langages, $n^{\circ} 136$, Sémantique lexicale et grammaticale , Paris, Larousse, 41-52.

MEL'ČUK, I. (dir.) $(1984,1988,1992,1999)$ : Dictionnaire explicatif et combinatoire du français contemporain : recherches lexico-sémantiques I, II, III, IV, Montréal, Presses universitaires de Montréal.

MEL'ČUK, I., CLAS, A. et POLGUÈRE, A. (1995) : Introduction à la lexicologie explicative et combinatoire, Louvain, Duculot.

MEL'ČUK, I. et WANNER, L. (1996) : Lexical Functions and Lexical Inheritance for Emotions Lexemes in German, in Lexical Functions in Lexicography and Natural Language Processing, Wanner, L. (ed.), Amsterdam, John Benjamins, 209-278.

MEL'ČUK, I. (1998) : Collocations and Lexical Functions, in Phraseology. Theory, Analysis and Applications, Cowie, A. P. (ed.), Oxford, Clarendon Press, 23-53.

TUTIN, A. et GROSSMANN, F. (2002) : Collocations régulières et irrégulières : esquisse de typologie du phénomène collocatif, Revue française de linguistique appliquée, 7-1, 7-25.

TUtin, A., NOVAKova, I., GROSSMANN, F. et CAVALLA, C. (à paraître) : Esquisse de typologie des noms d'affect à partir de leurs propriétés combinatoires, Langue française, Paris, Larousse.

Ressources électroniques

FRANTEXT : CNRS, ATILF (Analyse et traitement informatique de la langue française), UMR CNRSUniversité Nancy2. < http://atilf.atilf.fr/frantext.htm >. 


\section{NOTES}

1. Nous utiliserons ici la convention suivante : le terme SENTIMENT en majuscules désignera le générique, englobant les notions de sentiment et d'émotion que nous allons définir par la suite.

2. Cet article est le fruit d'un travail de recherche de maitrise et de master 2 traitant plus largement des collocations des N_SENT : GoosSENS, V. (2004) : L'organisation du champ sémantique des SENTIMENTS, TER de Maîtrise de Sciences du langage, dirigé par Grossmann, F., Grenoble, Université Stendhal-Grenoble 3. GoossENS, V. (2005) : Une typologie des noms de SENTIMENT basée sur leurs collocations verbales, mémoire de deuxième année de Master Descriptions linguistiques, dirigé par Grossmann, F., Grenoble, Université Stendhal-Grenoble 3.

3. Sur ce point voir également Jean-Claude Anscombre $(1995,1996)$ qui distingue les noms de sentiment et d'attitude " exogènes ", qui ont une cause extérieure au lieu psychologique, et les noms de sentiment et d'attitude « endogènes » chez qui l'origine se confond avec le lieu psychologique.

4. Nous nous sommes également appuyée sur d'autres travaux portant sur les N_SENT même s'ils ne traitaient pas du problème de la délimitation de cette classe : Bresson et Dobrovol'skij, 1995 ; Dirven, 1997 ; Gross, 1995 ; Grossmann et Tutin, à paraitre ; Leeman, 1991 ; Mathieu, 1999 ; Mel'čuk et Wanner, 1996.

5. Amour, angoisse, bonheur, chagrin, colère, compassion, crainte, dégoût, désespoir, effroi, ennui, envie, frayeur, fureur, gaieté, haine, honte, jalousie, joie, mélancolie, mépris, panique, peine, peur, rage, respect, stupeur, surprise, terreur, tristesse.

6. Nous avons consulté avec profit les travaux anciens sur les « passions » et les émotions (de Descartes à Darwin), pris en compte également la littérature récente qui nous a permis d'affiner certains de nos critères (par exemple Cosnier, 1994 ; Frijda, 1988).

7. Nous nous sommes limitée aux romans pour conserver une certaine homogénéité générique, à défaut de pouvoir couvrir de manière satisfaisante l'ensemble des genres.

8. Exemple de Hector Bianciotti, extrait de Frantext.

9. Exemple de Jean-Marie Gustave Le Clézio, extrait de Frantext.

10. Exemple de Julia Kristeva, extrait de Frantext.

11. Exemple de Hervé Bazin, extrait de Frantext.

12. Nous utilisons la convention de notation suivante : $X$ représente l'expérienceur/ agent, $\mathrm{Y}$ la cause et $\mathrm{Z}$ l'objet.

13. Une requête complémentaire sur Internet a permis de s'en assurer.

14. Nous ne traiterons pas ici ces collocations qui sont peu fréquentes dans notre corpus et ne concernent pas directement notre objet d'étude.

15. Le fait que stupeur, effroi, frayeur ou même surprise n'aient pas donné lieu à des collocations avec des verbes exprimant la possession peut sembler étonnant. Ce phénomène est peut-être lié à la taille du corpus et au faible nombre de collocations que certains de ces noms ont produit.

16. L'absence - surprenante - de causatifs avec angoisse est à vérifier sur un corpus plus vaste et plus diversifié. 


\section{AUTEUR}

\section{VANNINA GOOSSENS}

Vannina Goossens, Université Stendhal-Grenoble 3, Laboratoire Lidilem, E.A. 609. 